\title{
DOENÇAS ENDÓCRINAS, NUTRICIONAIS, METABÓLICAS E HÁBITOS ALIMENTARES EM ESTUDANTES ADOLESCENTES NOS ESTADOS E CAPITAIS DO BRASIL, 2019
}

\author{
Daniel Hideki Bando \\ Mariana Rangel Garcia² \\ Estevan $\mathrm{Coca}^{3}$
}

RESUMO: O presente estudo teve como objetivo analisar o padrão espacial das taxas de internação por doenças endócrinas, nutricionais, metabólicas em adolescentes e identificar associações com hábitos alimentares e estilo de vida dos escolares nos estados e capitais do Brasil. Dados foram extraídos do Sistema de Informações Hospitalares (SIH - DATASUS) e da Pesquisa Nacional de Saúde do Escolar (PeNSE - IBGE). Foram elaborados mapas coropléticos e as associações foram estimadas por meio de técnicas de correlação e regressão. Pernambuco e a capital Recife apresentaram as maiores taxas de internação, 8,0 e 11,1 por 10 mil habitantes, respectivamente. Em ordem decrescente, Paraná, Minas Gerais, Distrito Federal, Mato Grosso do Sul também apresentaram taxas elevadas, variando de 4,7 a 6,0 por 10 mil. Para as capitais, em ordem decrescente, Brasília, Belo Horizonte, Curitiba, Campo Grande apresentaram as maiores taxas, de 4,1 a 5,1 por 10 mil. Estados e capitais da macrorregião Norte apresentaram as menores taxas. O principal preditor para a taxa de internação por doenças endócrinas, nutricionais, metabólicas que permaneceu no modelo final com significancia estatística $(p<0,05)$ foi o consumo de alimentos ultraprocessados. A força da associação foi maior nas capitais.

PALAVRAS-CHAVE: internação; estilo de vida; alimento; geografia da saúde.

\section{ENDOCRINE, NUTRITIONAL AND METABOLIC DISEASES AND FOOD HABITS IN ADOLESCENT STUDENTS IN THE STATES AND CAPITALS OF BRAZIL, 2019}

ABSTRACT: This study aimed to analyze the spatial pattern of hospitalization rates for endocrine, nutritional and metabolic diseases in adolescents and to identify associations

\footnotetext{
1 Professor de Geografia da Universidade Federal de Alfenas; daniel.bando@unifal-mg.edu.br

2 Licenciada em Geografia pela Universidade Federal de Alfenas; mariana.rangel@sou.unifalmg.edu.br

3 Professor de Geografia da Universidade Federal de Alfenas; estevan.coca@unifal-mg.edu.br
} 
with eating habits and lifestyle of schoolchildren in the states and capitals of Brazil. Data were extracted from the Hospital Information System (SIH - DATASUS) and the National Survey of School Health (PeNSE - IBGE). Choropletic maps were drawn up and associations were estimated using correlation and regression techniques. Pernambuco and the capital Recife had the highest hospitalization rates, 8.0 and 11.1 per 10,000 inhabitants, respectively. In descending order, Paraná, Minas Gerais, Distrito Federal, Mato Grosso do Sul also presented high rates, ranging from 4.7 to 6.0 per 10,000. For the capitals, in descending order, Brasília, Belo Horizonte, Curitiba, Campo Grande had the highest rates, from 4.1 to 5.1 per 10,000. States and capitals of the North macro region had the lowest rates. The main predictor for the rate of hospitalization due to endocrine, nutritional and metabolic diseases that remained in the final model with statistical significance $(p<0.05)$ was the consumption of ultra-processed foods. The strength of the association was greater in the capitals.

KEYWORDS: hospitalization; Lifestyle; food; health geography

\section{ENFERMEDADES ENDOCRINAS, NUTRICIONALES, METABÓLICAS Y HÁBITOS ALIMENTARIOS EN ESTUDIANTES ADOLESCENTES EN LOS ESTADOS Y CAPITALES DE BRASIL, 2019}

RESUMEN: Este estudio tuvo como objetivo analizar el patrón espacial de las tasas de hospitalización por enfermedades endocrinas, nutricionales y metabólicas en adolescentes e identificar asociaciones con los hábitos alimentarios y el estilo de vida de los escolares en los estados y capitales de Brasil. Los datos se extrajeron del Sistema de Información Hospitalaria (SIH - DATASUS) y la Encuesta Nacional de Salud del Adolescente (PeNSE - IBGE). Se elaboraron mapas corpléticos y se estimaron las asociaciones mediante técnicas de correlación y regresión. Pernambuco y la capital Recife tuvieron las tasas de hospitalización más altas, 8,0 y 11,1 por 10 mil habitantes, respectivamente. En orden descendente, Paraná, Minas Gerais, Distrito Federal, Mato Grosso do Sul también presentaron tasas elevadas, que van de 4,7 a 6,0 por 10 mil. Para las capitales, en orden descendente, Brasilia, Belo Horizonte, Curitiba, Campo Grande tuvieron las tasas más altas, de 4,1 a 5,1 por 10 mil. Los estados y capitales de la macro región Norte tuvieron las tasas más bajas. El principal predictor de la tasa de hospitalización por enfermedades endocrinas, nutricionales y metabólicas que permaneció en el modelo final con significación estadística $(p<0,05)$ fue el consumo de alimentos ultraprocesados. La fuerza de la asociación fue mayor en las capitales.

PALABRAS CLAVE: hospitalización; estilo de vida; alimentos ultraprocesados; geografía de la salud 


\section{INTRODUÇÃO}

De acordo com a Pesquisa Nacional de Saúde (PNS) de 2019, o problema de saúde pública de maior magnitude atualmente são as doenças crônicas não transmissíveis (DCNT), pois além da mortalidade prematura, impactam diretamente na qualidade de vida dos doentes, da sociedade, na economia e no sistema de saúde (IBGE, 2020). Dentre as DCNT, destacam-se o grupo das doenças cardiovasculares (e.g. hipertensão, infarto), das doenças endócrinas, nutricionais e metabólicas (e.g. diabetes, obesidade, desnutrição) e das doenças mentais (e.g. depressão). Nas duas últimas décadas, as doenças cardiovasculares representam a maior carga de mortalidade no Brasil (28\%), já as mortes por doenças endócrinas, nutricionais e metabólicas correspondem a aproximadamente $6 \%$ (BANDO, 2018; DATASUS, 2021). Múltiplos fatores estão associados às DCNT, sendo que alguns são passíveis de modificação, como o hábito de fumar, de alimentação e prática de atividade física. O diabetes é considerado uma epidemia no mundo, a doença acometeu em 2014 mais de 422 milhões de pessoas no mundo, e em 2016, cerca de 1,6 milhão de pessoas morreram por causa direta da doença (OMS, 2021). Trata-se de uma das principais causas de cegueira, insuficiência renal, infarto, derrame e amputação de membros inferiores. A prevalência da obesidade e diabetes tem aumentado com maior velocidade em países de baixa e média renda como o Brasil (BRASIL, 2014).

No Brasil houve aumento progressivo do incentivo às indústrias alimentícias, o que acaba por gerar à população facilidade de acesso aos alimentos ultraprocessados (AUP) (MACHADO; OLIVEIRA; MENDES, 2016), caracterizados como formulações industriais ricas em sal, açúcar e hidrogenados, com baixo custo. Segundo Martins et al. (2013), a partir da década de 2000, o aumento de consumo de AUP ocorreu em todos os estratos de renda no país. A literatura tem evidenciado a associação entre o consumo de AUP com as DCNT, 
como a obesidade e diabetes (LANE; DAVIS; BEATTIE; GÓMEZ-DONOSO et al., 2021; SROUR; FEZEU; KESSE-GUYOT; ALLÈS et al., 2020). Há evidencias também do efeito protetor da educação alimentar adequada na prevenção de sobrepeso e obesidade em escolares (ARAÚJO; FERREIRA; NEUMANN; MIRANDA et al., 2017). Compreender a distribuição das DCNT e fatores associados no tempo e no espaço são fundamentais para o planejamento de políticas públicas e para a diminuição das desigualdades sociais.

A Pesquisa Nacional de Saúde do Escolar (PeNSE) surgiu a partir de uma parceria entre o Instituto Brasileiro de Geografia e Estatística (IBGE) e Ministério da Saúde (MS) com o apoio do Ministério da Educação (MEC), e tem como objetivo monitorar os fatores de risco e proteção à saúde dos adolescentes estudantes brasileiros, de escolas públicas e privadas (IBGE, 2021). Os indicadores da PeNSE contemplam diversos temas como hábitos alimentares, alimentação na escola, atividade física, saúde mental e imagem corporal, sendo fundamentais para o monitoramento e conhecimento dos agravos que acometem os alunos. Estudo baseado na primeira edição da PeNSE (2009) revelou consumo regular dos marcadores de alimentação não saudável e consumo inferior ao recomendado dos marcadores de alimentação saudável (LEVY; CASTRO; CARDOSO LDE; TAVARES et al., 2010). Com dados da segunda edição da PeNSE (2012), estudo identificou associação do consumo de alimentos ricos em açúcar com estudantes do sexo feminino, com maior escolaridade materna, que não viviam com a mãe e o pai, que não realizavam as refeições com os pais, que comiam assistindo TV e que passavam mais tempo diante da TV (FERREIRA; CLARO; LOPES, 2015). Estudo com dados da terceira edição da PeNSE (2015) identificou maior percentual de escolares com hábitos sedentários nos estados das regiões Sul e Sudeste, e uma correlação positiva e significativa dos mesmos com o consumo de AUP $(r=0,72$; $p<0,01)(G A R C I A ; B A N D O ; C O C A, 2021)$. 
O presente trabalho tem como objetivo analisar o padrão espacial das taxas de internação por doenças endócrinas, nutricionais, metabólicas em adolescentes e identificar possíveis associações com indicadores da PeNSE (2019), referentes a hábitos alimentares e estilo de vida.

\section{MATERIAIS E MÉTODOS}

Trata-se de um estudo exploratório, onde foram usados dados secundários agregados por Unidades da Federação (UF) e por capitais. As análises foram feitas para os dois grupos separadamente: UF e capitais. A taxa de internação por doenças endócrinas nutricionais e metabólicas foi definida como a variável dependente, ou resposta. Dados de internação hospitalar foram obtidos pelo Sistema de Informações Hospitalares (SIH), diretamente pelo Ministério da Saúde (DATASUS, 2021). O grupo das doenças endócrinas nutricionais e metabólicas correspondem ao capítulo IV da Décima Classificação Internacional de Doenças (CID-10), códigos “E00 a E90". Foram calculadas as taxas de internação (por 10 mil habitantes), em 2019, na faixa etária disponível mais próxima da utilizada pela PeNSE: de 10 a 19 anos. Foi utilizada a projeção da população estimada pelo IBGE para o respectivo ano e faixa etária.

As variáveis independentes, ou preditoras, foram os indicadores relacionados aos hábitos alimentares e estilo de vida dos escolares, de 13 a 17 anos, da quarta edição da PeNSE (2019) (IBGE, 2021), coletadas diretamente pelo site do IBGE. Foram selecionados indicadores de quatro temas. Todos indicadores representam o percentual (\%) de estudantes em determinada condição (Quadro 1).

Quadro 1 - Indicadores selecionados da PeNSE, 2019

\begin{tabular}{|c|c|}
\hline Tema & Indicador \\
\hline$\overline{\bar{\varangle}} \varepsilon$ & Consumiram bebida ultraprocessada no dia anterior, por tipo (5): refrigerante, suco de \\
\hline
\end{tabular}




\begin{tabular}{|c|c|}
\hline & fruta em caixinha, refresco em pó, achocolatado, iogurte com sabor \\
\hline & Consumiram 2 ou mais sobremesas industrializadas no dia anterior \\
\hline & $\begin{array}{l}\text { Consumiram alimento ultraprocessado salgado no dia anterior, por tipo (5): produto } \\
\text { cárneo, pão, margarina, molho, refeição pronta }\end{array}$ \\
\hline & Consumiram algum alimento ultraprocessado no dia anterior \\
\hline & $\begin{array}{l}\text { Consumiram alimento marcador de alimentação saudável cinco dias ou mais na } \\
\text { semana anterior (3): feijão, legumes/verduras, frutas }\end{array}$ \\
\hline & $\begin{array}{l}\text { Consumiram alimento marcador de alimentação não saudável cinco dias ou mais na } \\
\text { semana anterior (2): guloseimas doces, refrigerante }\end{array}$ \\
\hline & Costumavam tomar café da manhã cinco dias ou mais na semana \\
\hline & Faziam suas refeições concomitante às outras atividades \\
\hline & Cujas escolas ofereciam comida ou merenda escolar \\
\hline$\underset{\pi}{2 \pi} \frac{\pi}{0}$ & Consumiram comida ou merenda escolar oferecidos pela escola \\
\hline 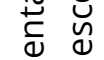 & Costumavam comprar alimentos ou bebidas na cantina dentro da escola \\
\hline 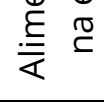 & $\begin{array}{l}\text { Costumavam comprar alimentos ou bebidas em ponto alternativo de venda (fora da } \\
\text { escola) }\end{array}$ \\
\hline & Com nenhum dia de aula de educação física na escola na semana anterior \\
\hline & Com 5 a 7 dias de aula de educação física na escola na semana anterior à pesquisa \\
\hline $\begin{array}{l}\frac{\pi}{0} \\
\frac{\pi}{0} . \underline{0}\end{array}$ & $\begin{array}{l}\text { Com } 300 \text { minutos ou mais de atividade física acumulada na semana anterior à } \\
\text { pesquisa }\end{array}$ \\
\hline$\sum_{i}^{\infty}$ & $\begin{array}{l}\text { Considerados inativos com base na atividade física acumulada na semana anterior à } \\
\text { pesquisa }\end{array}$ \\
\hline & Costumavam assistir televisão por mais de duas horas diariamente \\
\hline & Costumavam fazer atividades sentados mais de três horas diárias \\
\hline & Sentimento em relação ao próprio corpo: satisfeito, indiferente, insatisfeito \\
\hline & Autopercepção da imagem corporal: magro, normal, gordo \\
\hline & Atitude em relação ao próprio peso corporal: nenhuma, perder, ganhar, manter \\
\hline & Vomitaram ou tomaram laxantes para perder peso nos 30 dias anteriores \\
\hline$\stackrel{\widetilde{\sigma}}{\underline{\varepsilon}} \stackrel{0}{\circ}$ & $\begin{array}{l}\text { Ingeriram algum produto para perda de peso, sem acompanhamento nos } 30 \text { dias } \\
\text { anteriores }\end{array}$ \\
\hline & $\begin{array}{l}\text { Ingeriram algum produto para ganho de peso sem acompanhamento nos } 30 \text { dias } \\
\text { anteriores }\end{array}$ \\
\hline
\end{tabular}

Fonte: Pesquisa Nacional de Saúde do Escolar (PeNSE, 2019), IBGE 2021. Org. os autores

A análise espacial foi baseada nos dados por UF. Foram elaborados mapas coropléticos, com intervalos classificados pelo método de quebra natural com o programa ArcGIS 10.5. Para identificar associações entre as variáveis foram utilizadas correlações bivariadas de Pearson. As variáveis que apresentaram coeficientes de correlação significativos $(p<0,05)$ foram utilizadas para compor 0 modelo de regressão linear múltipla. Esse tipo de modelo é utilizado para estimar a relação entre uma variável dependente (y) e diversas variáveis independentes 
$\left(\mathrm{X}_{n}\right)$ (LONGLEY; GOODCHILD; MAGUIRE; RHIND, 2013). A técnica tenta modelar a relação entre as variáveis por meio de um ajuste de uma equação linear sobre os dados observados. Cada valor da variável independente é associado com o valor da variável dependente, depois de controlar por um número de covariáveis (e.g., $\left.x_{2}, x_{3} \ldots x_{n}\right)$. O modelo final é dado pela equação: $y=\beta_{0}+\beta_{1} x_{1}+\beta_{2} x_{2} \ldots \beta_{n} x_{n}$

O coeficiente de regressão $\left(\beta_{n}\right)$ corresponde à inclinação da linha de regressão e mede o sentido e a força da influência das variáveis independentes sobre a variável dependente. O $\beta_{0}$ é o intercepto, o valor onde a linha de regressão cruza o eixo Y. Para o modelo múltiplo final, foi utilizado o método por eliminação backward. O $\mathrm{R}^{2}$ ajustado, os coeficientes de regressão e respectivos níveis de significância foram utilizados para a escolha do modelo final. Os resíduos da regressão múltipla foram analisados quanto à normalidade usando o teste de Kolmogorov-Smirnov. Para verificar a presença de multicolinearidade foi utilizado o Fator de Inflação de Variância (FIV). Em todos os procedimentos de regressão, o valor de $p$ foi estabelecido em 0,05.

\section{RESULTADOS E DISCUSSÕES}

Em 2019, no Brasil, ocorreram 12.158 internações hospitalares por doenças endócrinas, nutricionais, metabólicas (Tabela 1), o que corresponde a uma taxa de internação de 3,7 por 10 mil habitantes. Mais da metade das internações foi por diabetes, seguido por outros transtornos endócrinos, depleção de volume, desnutrição, obesidade.

Tabela 1 - Internações por doenças endócrinas, nutricionais e metabólicas, na faixa etária dos 10 a 19 anos, no Brasil, por morbidade, 2019

\begin{tabular}{lcc}
\hline Doenças endócrinas, nutricionais e & Internações & $(\%)$ \\
\hline Diabetes mellitus & 6.611 & 54,38 \\
Outros transt endócrinos nutricionais & 3.219 & 26,48 \\
Depleção de volume & 1.206 & 9,92 \\
Desnutrição & 806 & 6,63
\end{tabular}




\begin{tabular}{lcc} 
Obesidade & 146 & 1,20 \\
Demais morbidades & 170 & 1,41 \\
Total & 12.158 & 100 \\
\hline
\end{tabular}

Fonte: DATASUS, 2019 Org. os autores

A coleção de mapas a seguir apresenta a distribuição espacial da variável dependente e os indicadores da PeNSE (Figura 1). Considerando-se as macrorregiões, a Sul (4,7 por 10 mil habitantes) e Sudeste (3,9 por 10 mil) apresentaram as maiores taxas de internação, a menor foi na Norte (1,8 por 10 mil). O estado de Pernambuco apresentou a maior taxa de internação por doenças endócrinas, nutricionais, metabólicas em estudantes, seguidos por Paraná, Minas Gerais, Distrito Federal e Mato Grosso do Sul (Figura 1A). Dentre as capitais, Recife apresentou a maior taxa (11,1 por 10 mil hab.), seguida por Brasília, Belo Horizonte e Curitiba. Algumas capitais da macrorregião Norte como Belém (0,46 por 10 mil), Rio Branco (0,74 por 10 mil) e do Nordeste como Natal, São Luís apresentaram as menores taxas. Com relação à incidencia por diabetes em adultos, a Pesquisa Nacional de Saúde de 2019, apresentou resultado condizente (IBGE, 2020). Segundo a pesquisa, o percentual da população que reportou diagnóstico médico de diabetes foi maior na região Sudeste $(8,5 \%)$ e Sul $(7,9 \%)$, e menor no Norte (5,5\%).

Quanto ao indicador "consumiram AUP no dia anterior (\%)" os valores foram elevados para todo o país (Figura 2B). São Paulo e Pernambuco apresentaram os maiores percentuais. É possivel notar agrupamentos com elevados percentuais formados por estados do Nordestede, Sul e Sudeste. Padrão semelhante foi descrito num estudo que usou dados da PeNSE de 2015 (GARCIA; BANDO; COCA, 2021). Nota-se relação direta desse mapa com o mapa das internações por doenças endócrinas (Figura 1A e 1B). Os AUP são projetados para maximizar o lucro, logo caracterizam-se pelos ingredientes de baixo custo, com longa validade, prontos para consumir (e.g. fast food) e palatáveis (MONTEIRO; 
CANNON; LEVY; MOUBARAC et al., 2019). Isso em parte explica o elevado consumo dos AUP em regiões carentes como o Nordeste. Ao mesmo tempo, sabe-se que na população adulta, o consumo dos AUP é maior em áreas urbanas (IBGE, 2020). Dados da PeNSE (2019) por capitais, retratam esses aspecto, em ordem decrescente, Recife, Fortaleza, Porto Alegre, Rio de Janeiro e São Paulo apresentaram as maiores proporções da população que consumiu AUP no dia anterior.

O indicador "consumiram refrigerante no dia anterior" concentrou as maiores proporções em ordem decrescente: São Paulo, Goiás e Distrito Federal (Figura 1C). Nota-se um agrupamento com elevado percentual formado por estados do Centro-Oeste $(45,9 \%)$, Sudeste $(45,1 \%)$ e Sul $(42,2 \%)$.

Figura 1 - Taxa de internação por doenças endócrinas nutricionais, metabólicas e indicadores da PenSE, por Unidade da Federação, 2019 

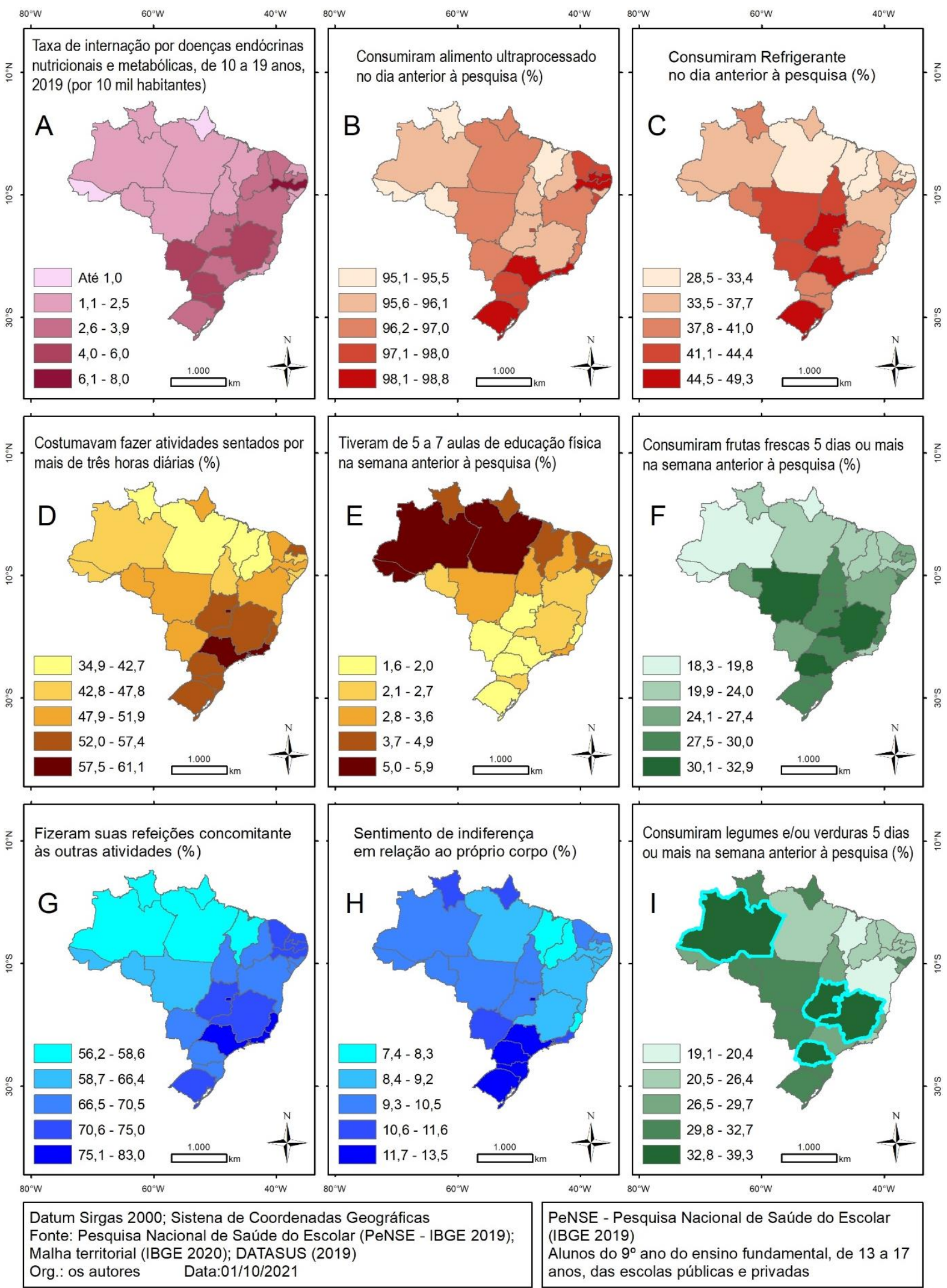

Estados do Norte e Nordeste apresentaram os menores percentuais. Nas capitais as maiores proporções foram em ordem decrescente: Cuiabá, Rio de 
Janeiro, Goiânia e Recife, variando de 48,8\% a 50,1\%. Logo, as capitais apresentaram maiores percentuais quando comparados com os estados. Para a população adulta, dados da Pesquisa Nacional de Saúde de 2019 apresentou resultados semelhantes. A "proporção de pessoas que consumiram refrigerante em pelo menos cinco dias da semana" foi maior nas regiões Centro-Oeste $(12,4 \%)$ e Sudeste (11,4\%), e a menor na Nordeste (5,2\%) (IBGE, 2020).

O indicador "costumavam fazer atividades sentados por mais de três horas diárias (\%)" apresentou maior proporção no Rio de Janeiro, São Paulo, Distrito Federal e estados vizinhos (Figura 1D). Padrão semelhante foi descrito num estudo com dados da PeNSE de 2015 (GARCIA; BANDO; COCA, 2021). Nas capitais os valores máximos foram um pouco acima dos estados, em ordem decrescente: Rio de Janeiro (64,3\%), Porto Alegre (63,3\%), São Paulo (62,0\%), Vitória (61,3\%). Estados das regiões Norte e Nordeste apresentaram os menores valores. A distribuição espacial do indicador "tiveram de 5 a 7 aulas de educação física na semana anterior à pesquisa (\%)" (Figura 1E) foi complementar ao mapa anterior (Figura 1D), com maiores percentuais na macrorregião Norte e Nordeste, e menores na Sul e Sudeste. Nas capitais o padrão foi semelhante, com exceção de Cuiabá. Em ordem decrescente os maiores percentuais foram em Rio Branco, Cuiabá, Manaus, Belém, variando de 4,0\% a 5,7\%.

Os maiores percentuais de alunos que "consumiram frutas frescas cinco dias ou mais na semana anterior" concentraram-se num agrupamento formado por estados da regiões Centro-Oeste (30,0\%), Sul (29,7\%) e Sudeste (28,1\%) (Figura 1F). As capitais seguiram padrão semelhante, as cinco capitais com o maior percentual correspondem aos cinco estados com maior percentual, mas com a ordem um pouco diferente: Belo Horizonte (33,8\%) Goiânia (33,3\%) Brasília $(32,9 \%)$ Curitiba $(31,9 \%)$ Florianópolis (31,5\%). Outro indicador de alimentação saudável, o "consumiram legumes e/ou verduras cinco dias ou mais na semana 
anterior", apresentou padrão semelhante ao indicador anterior, porém com a adição do estado do Amazonas (33,8\%, $4^{\text {a }}$ posição), compondo os cinco estados com maior percentual (Figura 11). Minas Gerais e sua capital Belo Horizonte apresentaram os maiores percentuais, 39,3\% e 43,1\%, respectivamente. Um dos fatores que ajudam a explicar esse padrão em Minas Gerais é o bom mecanismo de construção social de segurança alimentar com participação social na definição das políticas alimentares. Belo Horizonte também possui um bom sistema de abastecimento alimentar, que pode favorecer o consumo de frutas e legumes. Os menores percentuais, em ordem crescente, foram os estados do Maranhão, Bahia e Ceará. As respectivas capitais também apresentaram os menores percentuais, em ordem crescente foram Salvador, São Luís e Fortaleza.

Quanto ao indicador "fizeram suas refeições concomitante às outras atividades", nota-se um agrupamento com maiores proporções formado por estados do Sudeste (78,3\%) (Figura 1G). Nas capitais, as três maiores proporções também foram do Sudeste, em ordem decrescente foram Rio de Janeiro $(85,6 \%)$, Vitória (84,3\%), São Paulo (83,3\%). Destaca-se também que os percentuais das capitais apresentaram valores um pouco acima dos estados. $O$ "sentimento de indiferença em relação ao próprio corpo" concentrou os maiores percentuais na região Sul (13,0\%), seguido pelo Sudeste $(10,9 \%)$ e Centro-Oeste $(10,7 \%)$ (Figura 1H). As três capitais com a maior proporção do indicador coincidiram com os respectivos estados: Curitiba e Florianópolis (14,6\%), Porto Alegre (13,2\%). Já as capitais São Paulo e Belo Horizonte ficaram próximas à mediana Belém (12,0\%). Rio de Janeiro e Salvador ficaram entre as seis menores proporções, 10,5\% e 9,9\%, respectivamente.

A tabela a seguir apresenta os coeficientes de correlação de Pearson entre taxa de internação por doenças endócrinas, nutricionais, metabólicas e indicadores da PeNSE (Tabela 2). O hábito de "fazer as refeições concomitante às 
outras atividades" apresentou maior correlação nas UFs, nas capitais foi o "consumo de molho ultraprocessado", possivelmente relacionado aos fast food. Em segundo lugar, tanto para as UFs quanto para as capitais, foi o "consumo de alimento ultraprocessado no dia anterior", sendo que nas capitais o coeficiente foi um décimo acima e com valor de p menor que 0,01 . Vale ressaltar que nas capitais houve maior número de correlações com os alimentos ultraprocessados do que nos estados, cinco e três, respectivamente. Em parte isso pode ser explicado pelo fato da população residente em área urbana consumir o dobro de alimentos ultraprocessados em relação às áreas rurais (IBGE, 2020). Ademais, sabe-se que a introdução dos ultraprocessados na dieta brasileira teve seu início na década de 1980 nas áreas metropolitanas, disseminada para todo o país nas décadas seguintes (MARTINS; LEVY; CLARO; MOUBARAC et al., 2013).

Apenas o grupo das UFs apresentou associação positiva das taxas de internação com marcador de alimentação saudável (frutas frescas). Uma possível explicação deve-se à frutose, açúcar presente nas frutas (SARTORELLI; CARDOSO, 2006). Quanto ao tema "atividade física", apenas o grupo das UFs apresentou correlações, sendo positiva com o hábito de "fazer atividades sentados mais de três horas/dia" e negativa com "cinco a sete dias de aulas de educação física". Esse resultado sugere efeito protetor da atividade física e risco do sedentarismo para a ocorrência de doenças endócrinas, nutricionais e metabólicas. Nas capitais houve correlação positiva com o sentimento "indiferente em relação ao próprio corpo".

Tabela 2 - Coeficientes de correlação de Pearson entre taxa de internação por doenças endócrinas nutricionais e metabólicas e variáveis preditoras da PeNSE, nas UF e capitais, 2019

\begin{tabular}{|c|c|c|}
\hline & Variável & Coeficiente \\
\hline \multirow{3}{*}{\multicolumn{2}{|c|}{ 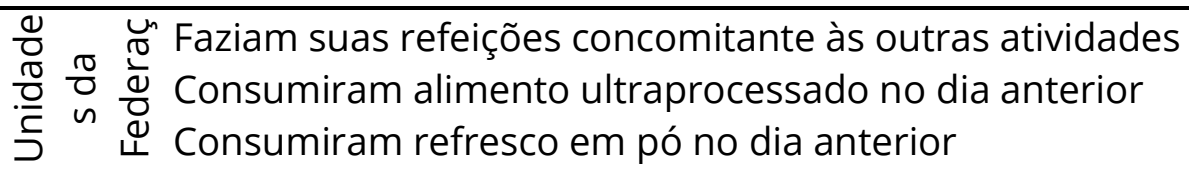 }} & $0,464^{*}$ \\
\hline & & $0,424^{*}$ \\
\hline & & 0,431 * \\
\hline
\end{tabular}


Costumavam fazer atividades sentados mais de três horas diárias

Consumiram frutas frescas cinco dias ou mais na semana anterior

Consumiram iogurte com sabor no dia anterior

Com 5 a 7 dias de aula de educação física na escola na semana

$-0,486$ * anterior

Consumiram alimento ultraprocessado salgado no dia da pesquisa: molho

Consumiram alimento ultraprocessado no dia anterior

Consumiram bebida ultraprocessada no dia anterior:

refrigerante

Consumiram alimento ultraprocessado salgado no dia da

pesquisa: carne

$0,420 *$

Consumiram merenda oferecidos pela escola na semana anterior: 1 a 2 dias

Sentimento em relação ao próprio corpo: indiferente

$0,404 *$

Consumiram 2 ou mais sobremesas industrializadas no dia anterior

$0,385^{*}$

${ }^{*} \mathrm{p}<0,05 ; *{ }_{p}<0,01$

A tabela a seguir apresenta os modelos de regressão múltipla (Tabela 3).

Para as UFs três indicadores permaneceram no modelo, sendo que apenas o "consumo de ultraprocessados" manteve a significância estatística. De acordo com o modelo, o aumento de $2 \%$ dos que "consumiram alimento ultraprocessado" corresponde ao aumento de uma internação em 10 mil habitantes. Para as capitais, dois indicadores permaneceram no modelo: "consumo de ultraprocessados" e "consumo de refrigerante". O aumento de $2 \%$ dos alunos que "consumiram alimento ultraprocessado" corresponde a um aumento de 2,3 internações em 10 mil habitantes. Já o aumento de $7 \%$ dos alunos que "consumiram refrigerante" corresponde ao acréscimo de uma internação em 10 mil habitantes. Portanto o "consumo de ultraprocessados" foi o principal preditor para a taxa de internação por doenças endócrinas nutricionais e metabólicas. Os resíduos das regressões apresentaram distribuição normal e o Fator de Inflação de Variância (FIV) não evidenciou presença de multicolinearidade em ambos 
modelos.

Tabela 3 - Resultado da regressão linear múltipla para o melhor conjunto de variáveis preditoras da PeNSE para taxa de internação por doenças endócrinas nutricionais e metabólicas nas UF e capitais

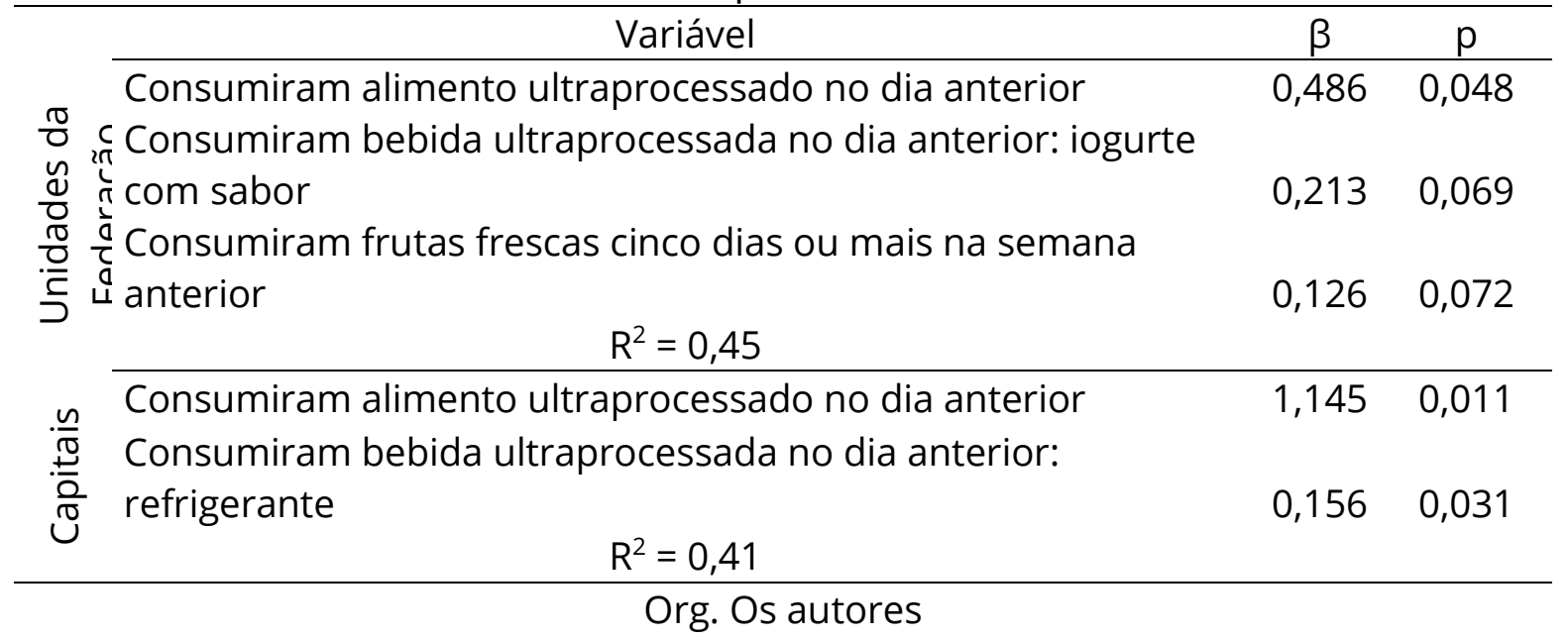

Os resultados do presente estudo reforçam os achados da literatura. Uma revisão sistemática e metanálise investigou a associação entre o consumo dos AUP com o risco de doenças crônicas não transmissíveis (DCNT), outras morbidades e também mortalidade. Foram incluídos 43 estudos observacionais, e o consumo de alimentos ultraprocessados foi associado ao aumento do risco de sobrepeso (Odds Ratio [OR]: 1,36), obesidade (OR: 1,51), obesidade abdominal (OR: 1,49), mortalidade por todas as causas (Risco Relativo [RR]: 1,28), síndrome metabólica (OR: 1,81), depressão (RR: 1,22), todos estatisticamente significativos. Além disso, o consumo de AUP foi associado a doenças cardiometabólicas, síndrome do intestino irritável, dispepsia funcional e câncer (de mama e geral) em adultos, além de síndrome metabólica em adolescentes e dislipidemia em crianças (LANE; DAVIS; BEATTIE; GÓMEZ-DONOSO et al., 2021). Outra metanálise conduzida por Schwingshackl et al., investigou a relação entre a ingestão de 12 grandes grupos de alimentos e o risco de diabetes tipo 2. Foram identificados como fatores de risco o consumo de carne vermelha e dos AUP como carne processada e bebidas adoçadas com açúcar (SCHWINGSHACKL; HOFFMANN; 
LAMPOUSI; KNÜPPEL et al., 2017).

Políticas públicas de prevenção da incidência de doenças endócrinas, nutricionais, metabólicas e demais DCNT em escolares, depende de vários setores como educação, saúde, agricultura e administração, com o objetivo de garantir uma cultura de alimentação e hábitos saudáveis (KROTH; GEREMIA; MUSSIO, 2020). É preciso valorizar a cultura alimentar e o modo de produção e consumo que incentivem os sistemas agroalimentares locais (TRICHES; PERONDI; FUHR, 2016). A revisão sistemática conduzida por Araújo et al., (2017) analisou o impacto das ações de educação alimentar e nutricional na prevenção de sobrepeso e obesidade entre escolares no período de 10 anos (2005-2015) no país. O resultado apontou que as ações de educação alimentar apresentaram impacto positivo na prevenção do sobrepeso e obesidade, portanto, essas ações precisam de incentivos no país.

\section{CONSIDERAÇÕES FINAIS}

O presente estudo identificou o padrão espaciais das taxas de internação por doenças endócrinas, nutricionais, metabólicas em adolescentes. As regiões Sul e Sudeste apresentaram as maiores taxas, a Norte a menor. Por estado, Pernambuco apresentou a maior taxa, seguido por Paraná e Minas Gerais. Quanto ao consumo de AUP, São Paulo e Pernambuco apresentaram os maiores percentuais. Foi possível observar agrupamentos com maiores percentuais formados por estados do Nordeste, Sul e Sudeste. O consumo de AUP foi o principal preditor associadodo às taxas de internação por doenças endócrinas, nutricionais, metabólicas. Esses padrões merecem uma investigação aprofundada, em grupos específicos, por exemplo, estratificados por sexo e idade. A PeNSE também dispõe os indicadores separados por escolas públicas e privadas, além de outros temas que não foram abordados nesse estudo. 
A última edição da PeNSE foi realizada em 2019, imediatamente antes da pandemia de COVID-19. Passamos a conviver com estilos de vida diferentes, como o isolamento social, eventos online, sedentarismo, mudança de hábitos alimentares. Portanto a PeNSE cumprirá um papel primordial, de avaliar o impacto da pandemia nos fatores de risco e proteção à saúde dos escolares. Mas para isso, é preciso que a pesquisa continue. Vivemos em tempos sombrios, de crise sanitária, política, econômica e ambiental sem precedentes. Presenciamos o genocídio de mais de 612 mil pessoas pela COVID-19, o aumento do desemprego e da fome que voltaram a assolar o país. Houve cortes de recursos na educação, em ciência/tecnologia, cortes no orçamento do IBGE que comprometem o próximo censo demográfico e a próxima edição da PeNSE. Diante disso, não podemos recuar, nem perder a esperança, é preciso manter a chama da luta acesa. Nas palavras de Paulo Freire (1997) “Ela [a esperança], só, não ganha a luta, mas sem ela a luta fraqueja e titubeia. Precisamos de herança crítica, como o peixe precisa da água despoluída".

\section{REFERÊNCIAS BIBLIOGRÁFICAS}

ARAÚJO, A. L.; FERREIRA, V. A.; NEUMANN, D.; MIRANDA, L. S. et al. O impacto da educação alimentar e nutricional na prevenção do excesso de peso em escolares: uma revisão bibliográfica. RBONE - Revista Brasileira de Obesidade, Nutrição e Emagrecimento, 11, n. 62, p. 11, 02/12 2017.

BANDO, D. H. Geografia da saúde mineira. In: VALE, A. R. d. e FERREIRA, M. M. F. (Ed.). Dinâmicas Geográficas no Sul de Minas Gerais. Curitiba: Appris, 2018. p. 4978.

BRASIL. Ministério da Saúde. Secretaria de Atenção à Saúde. Departamento de Atenção Básica. Guia alimentar para a população brasileira. Brasília: Ministério da Saúde, 2014. 158 p. 978-85-334-2176-9. 
DATASUS. Departamento de Informática do Sistema Único de Saúde. Ministério da Saúde. 2021. Disponível em: http://www2.datasus.gov.br/DATASUS/index.php.

FERREIRA, N. L.; CLARO, R. M.; LOPES, A. C. Consumption of sugar-rich food products among Brazilian students:National School Health Survey (PeNSE 2012). Cad Saude Publica, 31, n. 12, p. 2493-2504, Dec 2015.

FREIRE, P. Pedagogia da esperança: Um reencontro com a pedagogia do oprimido. Rio de Janeiro: Paz e Terra, 1997. 336 p. 9788577532278.

GARCIA, M. R.; BANDO, D. H.; COCA, E. L. Padrões espaciais de indicadores de hábitos alimentares e de saúde em adolescentes no Brasil (2015). In: X Simpósio Nacional de Geografia da Saúde: dimensões geográficas dos impactos e desafios das pandemias, 2021, Campina Grande. p. 514-526. Anais. Disponível em: https://www.anaisgeosaude.com.

IBGE. Instituto Brasileiro de Geografi a e Estatística. Pesquisa Nacional de Saúde 2019. Percepção do estado de saúde, estilos de vida, doenças crônicas e saúde bucal: Brasil e grandes regiões. Rio de Janeiro: IBGE, 2020. 978-65-87201-33-7.

IBGE. Instituto Brasileiro de Geografia e Estatística. Pesquisa Nacional de Saúde do Escolar: 2019. Rio de Janeiro: IBGE, 2021. 978-65-87201-77-1.

KROTH, D. C.; GEREMIA, D. S.; MUSSIO, B. R. Programa Nacional de Alimentação Escolar: uma política pública saudável. Cien Saude Colet, 25, n. 10, p. 4065-4076, Oct 2020.

LANE, M. M.; DAVIS, J. A.; BEATTIE, S.; GÓMEZ-DONOSO, C. et al. Ultraprocessed food and chronic noncommunicable diseases: A systematic review and metaanalysis of 43 observational studies. Obes Rev, 22, n. 3, p. e13146, Mar 2021.

LEVY, R. B.; CASTRO, I. R.; CARDOSO LDE, O.; TAVARES, L. F. et al. Consumo e comportamento alimentar entre adolescentes brasileiros: Pesquisa Nacional de Saúde do Escolar (PeNSE), 2009. Cien Saude Colet, 15 Suppl 2, p. 3085-3097, Oct 2010.

LONGLEY, P. A.; GOODCHILD, M. F.; MAGUIRE, D. J.; RHIND, D. W. Sistemas e Ciência da Informação Geográfica. Porto Alegre: Bookman, 2013. 9788565837651. MACHADO, P. P.; OLIVEIRA, N. R. F. d.; MENDES, A. N. O indigesto sistema do alimento mercadoria. Saúde e Sociedade, 25, n. 2, p. 505-515, 2016. 
MARTINS, A. P. B.; LEVY, R. B.; CLARO, R. M.; MOUBARAC, J. C. et al. Participação crescente de produtos ultraprocessados na dieta brasileira (1987-2009). Revista de Saúde Pública, 47, n. 4, p. 656-665, 2013.

MONTEIRO, C. A.; CANNON, G.; LEVY, R. B.; MOUBARAC, J. C. et al. Ultra-processed foods: what they are and how to identify them. Public Health Nutr, 22, n. 5, p. 936941, Apr 2019.

OMS. Organização Mundial da Saúde. Diabetes factsheet. 2021. Disponível em: https://www.who.int/news-room/fact-sheets/detail/diabetes.

SARTORELLI, D. S.; CARDOSO, M. A. Associação entre carboidratos da dieta habitual e diabetes mellitus tipo 2: evidências epidemiológicas. Arq Bras Endocrinol Metabol, 50, n. 3, p. 415-426, Jun 2006.

SCHWINGSHACKL, L.; HOFFMANN, G.; LAMPOUSI, A. M.; KNÜPPEL, S. et al. Food groups and risk of type 2 diabetes mellitus: a systematic review and meta-analysis of prospective studies. Eur J Epidemiol, 32, n. 5, p. 363-375, May 2017.

SROUR, B.; FEZEU, L. K.; KESSE-GUYOT, E.; ALLÈS, B. et al. Ultraprocessed Food Consumption and Risk of Type 2 Diabetes Among Participants of the NutriNetSanté Prospective Cohort. JAMA Intern Med, 180, n. 2, p. 283-291, Feb 12020.

TRICHES, R. M.; PERONDI, C.; FUHR, A. L. Educação alimentar para sistemas agroalimentares locais no contexto da alimentação escolar. Revista Faz Ciência, 17, n. 26, p. 50, 08/22 2016. 